\title{
An Investor's Investment Plan with Stochastic Interest Rate under the CEV Model and the Ornstein-Uhlenbeck Process
}

\author{
Edikan E. Akpanibaha, ${ }^{\mathrm{a}, *}$ Udeme O. Ini ${ }^{\mathrm{b}}$ \\ ${ }^{a}$ Department of Mathematics and Statistics, Federal University Otuoke, Bayelsa, Nigeria \\ ${ }^{b}$ Department of Mathematics and Computer Science, Niger Delta University, Bayelsa, Nigeria
}

\begin{abstract}
The aim of this paper is to maximize an investor's terminal wealth which exhibits constant relative risk aversion (CRRA). Considering the fluctuating nature of the stock market price, it is imperative for investors to study and develop an effective investment plan that considers the volatility of the stock market price and the fluctuation in interest rate. To achieve this, the optimal investment plan for an investor with logarithm utility under constant elasticity of variance (CEV) model in the presence of stochastic interest rate is considered. Also, a portfolio with one risk free asset and two risky assets is considered where the risk free interest rate follows the Ornstein-Uhlenbeck (O-U) process and the two risky assets follow the CEV process. Using the Legendre transformation and dual theory with asymptotic expansion technique, closed form solutions of the optimal investment plans are obtained. Furthermore, the impacts of some sensitive parameters on the optimal investment plans are analyzed numerically. We observed that the optimal investment plan for the three assets give a fluctuation effect, showing that the investor's behaviour in his investment pattern changes at different time intervals due to some information available in the financial market such as the fluctuations in the risk free interest rate occasioned by the O-U process, appreciation rates of the risky assets prices and the volatility of the stock market price due to changes in the elasticity parameters. Also, the optimal investment plans for the risky assets are directly proportional to the elasticity parameters and inversely proportional to the risk free interest rate and does not depend on the risk averse coefficient.
\end{abstract}

DOI: $10.46481 /$ jnsps.2021.172

Keywords: O-U process, Stochastic interest rate, Optimal investment plan, Legendre transform, CEV model

\section{Article History :}

Received: 13 March 2021

Received in revised form: 27 May 2021

Accepted for publication: 07 June 2021

Published: 29 August 2021

(c)2021 Journal of the Nigerian Society of Physical Sciences. All rights reserved. Communicated by: T. Latunde

\section{Introduction}

In the study of the optimal investment plan for any financial institution and considering the fact that the economy of most countries and even the financial markets are presently in serious crisis due to the suffocating impact of the novel Covid-19 pandemic, the role of stochastic volatilities cannot be over emphasized as it plays a significant role in the behaviour of the

${ }^{*}$ Corresponding author tel. no: +2347030811189

Email address: edemae@fuotuoke.edu.ng (Edikan E. Akpanibah ) stock market price due to its unstable nature resulting from various information obtainable from the market. In order to take a seemingly right decision while investing in risky assets such as stock, the stochastic volatility models become necessary to understand the random nature of the stock market price. In this paper, we consider the CEV model which is one of the stochastic volatility models used in describing the behaviour of the stock market price. This model was first used in [2] and degenerate into a constant volatility model called the geometric Brownian motion (GBM) when its elasticity parameter equals zero. One significant property of this model is its ability to capture the 
implied volatility skew.

Several works have been done on utility maximization under the CEV model, some of which include [3] - [5]. In [6], the reinsurance problem and optimal investment under the CEV model was studied. [1], studied the optimal investment problem with taxes, dividend and transaction cost using different utility functions under the CEV process. [7, 8] solved the optimal investment problem for a defined contribution (DC) pension plan with return of premiums clauses under different assumptions and assumed that the stock market price follows the CEV process; they used the game theoretic approach and solved the resultant extended Hamilton Jacobi Bellman equation for the optimal investment plan. The optimal investment plan with stochastic interest rate under GBM has been studied by some authors; these include [9], who studied the investment portfolio under stochastic interest rate for a case of protected DC fund. Also, [10, 11], used stochastic interest rate to obtain optimal investment plan for a DC plan. In $[12,13]$, the optimal investment plan with the Vasicek interest rate was studied while [14] - [16] solved some optimization problems for the optimal investment plan when the interest rate is of affine type.

The optimal investment plan with the CEV process under several assumptions has been studied by different authors when the risk free interest rate are constant as seen in the above literatures except for [17] - [19], who investigated the optimal control strategies under the CEV model with stochastic interest rate. In [17], the optimal investment plan of an insurer with stochastic interest rate under the CEV model was studied using logarithm utility, they assumed that the risk free interest rate follows the Cox- Ingersoll-Ross (CIR) process and used the power transformation, change of variable and asymptotic approach to find the asymptotic solution of the optimal investment plan. In [18], the expected utility of an investor with exponential utility function was maximized using the Legendre transformation and asymptotic expansion method to find a closed form solution of the optimal investment plan. They pointed out that authors find it difficult to combine the CEV model and stochastic interest rate in determining investment strategies due to the complexity of the resultant optimization problem. Also, they pointed out that in real life applications, interest rates are usually not constant but fluctuating in nature and the volatility of the interest rate generate some market risks; that is to say, when this risk are not considered, we are under estimating the effect of this risk emanating from this interest rate which is critical in influencing the prices of different assets available in financial market. In [19], the optimal investment plan for an investor with exponential utility under the modified CEV model was studied. In their work, the risk free rate follows the O-U process. Most of the literatures mentioned above used the (CIR) model, Vasicek model, affine model etc. to model their interest rate, however very few used O-U process. According to [21], we know that it is more accurate to use $\mathrm{O}-\mathrm{U}$ process in modelling both interest rate and stock market price since it reflects the fluctuation of the interest rates and asset prices. Also, the $\mathrm{O}-\mathrm{U}$ process is closer to the change in interest rate.

Based on this important characteristic of the O-U process and considering the unstable nature of the financial market at this present time, we are motivated to develop a robust investment plan for an investor with logarithm utility which takes into considerations fluctuations in interest rate as well as the volatility of the stock market prices. We do this by studying the optimal investment plans of an investor exhibiting the CRRA and whose risky assets and risk free interest rate are modelled by the CEV process and the O-U process respectively. Furthermore, the Legendre transformation and asymptotic technique are used to determine asymptotic solutions of the optimal investment plan. We also give some numerical simulations to explain our results. The main difference between our work and that of [18] is that we will consider an investor with logarithm utility where the investor's behaviour toward risks are not influenced by the risk aversion coefficient instead of the exponential utility which depend on the risk aversion coefficient. Also, investment is done with three assets instead of two assets and the risk free interest rate follows the $\mathrm{O}-\mathrm{U}$ process instead of the CIR process. The rest of the paper is outlined as follows; Section 2 described the financial models of the three assets and the risk free rate. Section 3 gives the definition of the value function, derives the corresponding Hamilton Jacobi Bellman equations by maximum principle and application of Legendre transform to the HJB equation. Section 4 provides closed-form solutions of the optimal investment plan for the three assets under CRRA utility function. Section 5 and 6 present numerical simulations of the effects of some sensitive parameters on the optimal investment plan and discussions. Section 7 concludes the work.

\section{The Financial Market Model}

Consider a portfolio comprising of one risk free asset and two risky assets in a financial market which is open continuously for an interval $t \in[0, T]$ where $T$ is the expiration date of the investment. Let $\left\{\mathcal{B}_{0}(t), \mathcal{B}_{1}(t), \mathcal{B}_{2}(t): t \geq 0\right\}$ be standard Brownian motion defined on a complete probability space $(\Omega, F, P)$ where $\Omega$ is a real space and $P$ is a probability measure and $F$ is the filtration which represents the information generated by the three Brownian motions.

Let $\mathcal{S}_{t}(t)$ denote the price of the risk free asset at time $t$ and the model is given as follows

$$
\frac{d \mathcal{S}_{0}(t)}{\mathcal{S}_{0}(t)}=r(t) d t \mathcal{S}_{0}(0)=s_{0}>0
$$

where $r(t)$ is the short interest rate process and driven by the stochastic differential equation

$$
d r(t)=\theta\left(\mu_{0}-r(t)\right) d t-\delta d \mathcal{B}_{0}(t), r(0)=r_{0}>0
$$

where $\theta, \mu_{0}$ and $\delta$ are positive real numbers see [19] - [21]. Let $\mathcal{S}_{1}(t)$ and $\mathcal{S}_{2}(t)$ denote the prices of two different stocks which are described by the CEV model and the dynamics of the stock market prices are described by the stochastic differential equations at $t \geq 0$ as follows

$$
\begin{aligned}
& \frac{d \mathcal{S}_{1}(t)}{\mathcal{S}_{1}(t)}=\mu_{1} d t+\sigma_{11} \mathcal{S}_{1}^{\beta}(t) d \mathcal{B}_{1}(t)+\sigma_{12} \mathcal{S}_{1}^{\beta}(t) d \mathcal{B}_{2}(t)(3) \\
& \frac{d \mathcal{S}_{2}(t)}{\mathcal{S}_{2}(t)}=\mu_{2} d t+\sigma_{21} \mathcal{S}_{2}^{\beta}(t) d \mathcal{B}_{1}(t)+\sigma_{22} \mathcal{S}_{2}^{\beta}(t) d \mathcal{B}_{2}(t)(4)
\end{aligned}
$$


where $\mu_{1}$ and $\mu_{2}$ are appreciation rate of the two risky assets, $\sigma_{11} \mathcal{S}_{1}^{\beta}, \sigma_{12} \mathcal{S}_{1}^{\beta}, \sigma_{21} \mathcal{S}_{2}^{\beta}$ and $\sigma_{22} \mathcal{S}_{2}^{\beta}$ are instantaneous volatilities and form a $2 \times 2$ matrix $\sigma=\left\{\sigma_{m n}\right\}_{2 \times 2}$ such that $\sigma \sigma^{T}$ is positive definite and $\beta<0$ represent elasticity parameter. (see [20] for details). Note if $\beta=0$, the stock market price is modelled by GBM.

\section{Optimization Problem}

\subsection{Wealth Formulations and HJB Equation}

Let $\varphi$ be the optimal portfolio strategy and we define the utility attained by the investor from a given state $x$ at time $t$ as

$$
\begin{aligned}
& \mathcal{L}_{\varphi}\left(t, r, s_{1}, s_{2}, x\right)= \\
& E_{\varphi}\left[\begin{array}{c|c}
U(X(T))=r, \\
\mathcal{S}_{1}(t)=s_{1}, \mathcal{S}_{2}(t)=s_{2}, \mathcal{X}(t)=x
\end{array}\right],
\end{aligned}
$$

where $t$ is the time, $r$ is the risk free interest rate and $x$ is the wealth.

The objective here is to determine the optimal portfolio strategies $\varphi^{*}=\left(\varphi_{0}, \varphi_{1}, \varphi_{2}\right)$ and the optimal value function of the investor $\mathcal{L}\left(t, r, s_{1}, s_{2}, x\right)$ given as

$$
\mathcal{L}\left(t, r, s_{1}, s_{2}, x\right)=\sup _{\varphi^{*}} \mathcal{L}_{\varphi^{*}}\left(t, r, s_{1}, s_{2}, x\right)
$$

such that

$$
\mathcal{L}_{\varphi^{*}}\left(t, r, s_{1}, s_{2}, x\right)=\mathcal{L}\left(t, r, s_{1}, s_{2}, x\right) .
$$

Let $\mathcal{X}(t)$ be the investor's wealth at time $t$ and the differential form associated with the fund size is given by

$$
\mathrm{d} X(t)=\mathcal{X}(t)\left(\varphi_{0} \frac{d \mathcal{S}_{0}(t)}{\mathcal{S}_{0}(t)}+\varphi_{1} \frac{d \mathcal{S}_{1}(t)}{\mathcal{S}_{1}(t)}+\varphi_{2} \frac{d \mathcal{S}_{2}(t)}{\mathcal{S}_{2}(t)}\right)
$$

Substituting (1), (3) and (4) into (8), we have

$$
\mathrm{d} \mathcal{X}(t)=\mathcal{X}(t)\left(\begin{array}{c}
\left(\varphi_{1}\left(\mu_{1}-r\right)+\varphi_{2}\left(\mu_{2}-r\right)+r\right) d t \\
+\left(\varphi_{1} \sigma_{11} \mathcal{S}_{1}^{\beta}(t)+\varphi_{2} \sigma_{21} \mathcal{S}_{2}^{\beta}(t)\right) d \mathcal{B}_{1} \\
+\left(\varphi_{1} \sigma_{12} \mathcal{S}_{1}^{\beta}(t)+\varphi_{2} \sigma_{22} \mathcal{S}_{2}^{\beta}(t)\right) d \mathcal{B}_{2} \\
\mathcal{X}(0)=\mathcal{X}_{0}
\end{array}\right)
$$

where $\varphi_{0}, \varphi_{1}$ and $\varphi_{2}$ are the optimal investment plans for the risk-free asset and the two risky assets respectively, such that $\varphi_{0}=1-\varphi_{1}-\varphi_{2}$.

From [19], applying the Ito's lemma and maximum principle, the Hamilton Jacobi Bellman (HJB) equation which is a nonlin-
Differentiating (10) with respect to $\varphi_{1}$ and $\varphi_{2}$, we obtain the first order maximizing condition for equation (10) as

$$
\begin{aligned}
& \varphi_{1}^{*}=\left(\begin{array}{c}
-\frac{\left[\mathcal{C} s_{1}^{\beta}\left(\mu_{2}-r\right)-\mathcal{B} s_{2}^{\beta}\left(\mu_{1}-r\right)\right]}{x\left(\mathcal{A B}-C^{2}\right) s_{1}^{2 \beta} s_{2}^{\beta}} \frac{\mathcal{L}_{x}}{\mathcal{L}_{x x}}-s_{1} \frac{\mathcal{L}_{x s_{1}}}{x \mathcal{L}_{x x}} \\
-\frac{\left(\mathcal{B D}-\mathcal{C}^{\mathcal{E}}\right) \delta \rho}{x\left(\mathcal{A B}-C^{2}\right) s_{1}^{\beta}} \frac{\mathcal{L}_{x x}}{\mathcal{L}_{x x}}
\end{array}\right) \\
& \varphi_{2}^{*}=\left(\begin{array}{c}
-\frac{\left[\mathcal{C} s_{2}^{\beta}\left(\mu_{1}-r\right)-\mathcal{A} s_{1}^{\beta}\left(\mu_{2}-r\right)\right]}{x\left(\mathcal{A B}-C^{2}\right) s_{1}^{\beta} s_{2}^{2 \beta}} \frac{\mathcal{L}_{x}}{\mathcal{L}_{x x}}-s_{2} \frac{\mathcal{L}_{x s_{2}}}{x \mathcal{L}_{x x}} \\
-\frac{(\mathcal{A} \mathcal{L}-\mathcal{C} \mathcal{D}) \delta \rho}{x\left(\mathcal{A B} \mathcal{B}-C^{2}\right) s_{2}^{\beta}} \frac{\mathcal{L}_{x x}}{\mathcal{L}_{x x}}
\end{array}\right)
\end{aligned}
$$

Substituting (11) and (12) into (10), we have

$$
\begin{aligned}
& \mathcal{L}_{t}+\mu_{1} s_{1} \mathcal{L}_{s_{1}}+\mu_{2} s_{2} \mathcal{L}_{s_{2}}+\frac{1}{2} \mathcal{A} s_{1}^{2 \beta+2} \mathcal{L}_{s_{1} s_{1}} \\
& +\frac{1}{2} \mathcal{B} s_{2}^{2 \beta+2} \mathcal{L}_{s_{2} s_{2}}+C s_{1}^{\beta+1} s_{2}^{\beta+1} \mathcal{L}_{s_{1} s_{2}} \\
& +\theta\left(\mu_{0}-r\right) \mathcal{L}_{r}+\frac{1}{2} \delta^{2} J_{r r}+\mathcal{D} \delta \rho s_{1}^{\beta+1} \mathcal{L}_{s_{1} r} \\
& +\mathcal{E} \delta \rho s_{2}^{\beta+1} \mathcal{L}_{s_{2} r}+\frac{1}{2}\left(\frac{\mathcal{F}}{s_{1}^{\beta} s_{2}^{\beta}}-\frac{\mathcal{G}}{s_{1}^{2 \beta}}-\frac{\mathcal{H}}{s_{2}^{2 \beta}}\right) \frac{\mathcal{L}_{x}^{2}}{\mathcal{L}_{x x}} \\
& \left.+r x \mathcal{L}_{x}-\left(\mu_{1}-r\right) s_{1} \frac{\mathcal{L}_{x} \mathcal{L}_{x s_{1}}^{1}}{\mathcal{L}_{x x}}-\left(\mu_{2}-r\right) s_{2} \frac{\mathcal{L}_{x} \mathcal{L}_{x s_{2}}}{\mathcal{L}_{x x}}\right\}=0(13) \\
& -\delta \rho\left(\frac{k_{1}\left(\mu_{1}-r\right)}{s_{1}^{\beta}}+\frac{k_{2}\left(\mu_{2}-r\right)}{s_{2}^{\beta}}\right) \frac{\mathcal{L}_{x} \mathcal{L}_{x r}}{\mathcal{L}_{x x}} \\
& -\frac{1}{2} \mathcal{A} s_{1}^{2 \beta+2} \frac{\mathcal{L}_{x s_{1}}^{2}}{\mathcal{L}_{x x}}-\frac{1}{2} \mathcal{B} s_{2}^{2 \beta+2} \frac{\mathcal{L}_{x s_{2}}^{2}}{\mathcal{L}_{x x}} \\
& -\frac{1}{2} k_{3} \rho^{2} \delta^{2} \frac{\mathcal{L}_{x r}^{2}}{\mathcal{L}_{x x}}-C s_{1}^{\beta+1} s_{2}^{\beta+1} \frac{\mathcal{L}_{x s_{1} \mathcal{L}_{x s_{2}}}}{\mathcal{L}_{x x}} \\
& \left\{\begin{array}{c}
\mathcal{A}=\sigma_{11}^{2}+\sigma_{12}^{2}, \quad \mathcal{B}=\sigma_{21}^{2}+\sigma_{22}^{2}, \\
\mathcal{C}=\sigma_{11} \sigma_{21}+\sigma_{12} \sigma_{22}, \mathcal{D}=\sigma_{11}+\sigma_{12}, \mathcal{E}=\sigma_{21}+\sigma_{22}
\end{array}\right. \\
& \mathcal{F}=\frac{2 C\left(\mu_{1}-r\right)\left(\mu_{2}-r\right)}{\left(\mathcal{A B}-C^{2}\right)}, \mathcal{G}=\frac{\mathcal{B}\left(\mu_{1}-r\right)^{2}}{\left(\mathcal{A B}-C^{2}\right)}, \mathcal{H}=\frac{\mathcal{A}\left(\mu_{2}-r\right)^{2}}{\left(\mathcal{A B}-C^{2}\right)}, \\
& k_{1}=\frac{(\mathcal{B D}-C \mathcal{E})}{\left(\mathcal{A B}-C^{2}\right)}, k_{2}=\frac{(\mathcal{A B}-C \mathcal{D})}{\left(\mathcal{A B}-C^{2}\right)} \cdot k_{3}=\frac{\left(\mathcal{B D}^{2}+\mathcal{A} \mathcal{E}^{2}-2 C \mathcal{D E}\right)}{\left(\mathcal{A B}-C^{2}\right)}
\end{aligned}
$$

where

From [20], we assumed that the optimal investment plan for risky assets' prices are known based on the assumption that

$$
\mu_{1} \varphi_{1}^{*}+\mu_{2} \varphi_{2}^{*}=n
$$

where $n$ is a constant.

Substituting (11) and (12) into (15), we derive an expression for $\frac{1}{s_{1}^{\beta} s_{2}}$ as

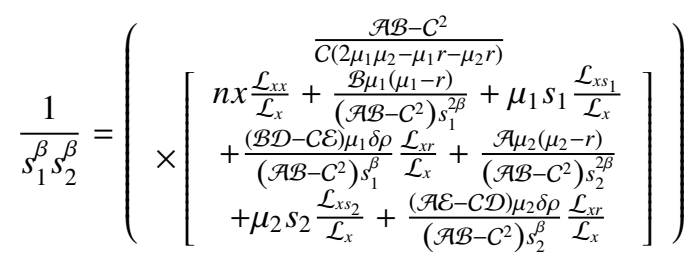

Substituting (16) into (13), we have subject to the investor's wealth as follows

$$
\left\{\begin{array}{c}
\mathcal{L}_{t}+\mu_{1} s_{1} \mathcal{L}_{s_{1}}+\mu_{2} s_{2} \mathcal{L}_{s_{2}}+\frac{1}{2} \mathcal{A} s_{1}^{2 \beta+2} \mathcal{L}_{s_{1} s_{1}} \\
+\frac{1}{2} \mathcal{B} s_{2}^{2 \beta+2} \mathcal{L}_{s_{2} s_{2}}+C s_{1}^{\beta+1} s_{2}^{\beta+1} \mathcal{L}_{s_{1} s_{2}} \\
+r x \mathcal{L}_{x}+\theta\left(\mu_{0}-r\right) \mathcal{L}_{r} \\
+\frac{1}{2} \delta^{2} \mathcal{L}_{r r}+\mathcal{D} \delta \rho s_{1}^{\beta+1} \mathcal{L}_{s_{1} r}+\mathcal{E} \delta \rho s_{2}^{\beta+1} \mathcal{L}_{s_{2} r} \\
+\sup _{\varphi_{1}, \varphi_{2}}\left\{\begin{array}{c}
\frac{1}{2} \mathcal{A} \varphi_{1}^{2} s_{1}^{2 \beta}+C \varphi_{1} \varphi_{2} s_{1}^{\beta} s_{2}^{\beta} \\
+\frac{1}{2} \mathcal{B} \varphi_{2}^{2} s_{2}^{2 \beta} \mathcal{L}_{x x} \\
\left(\left(\mu_{1}-r\right) \varphi_{1}+\left(\mu_{2}-r\right) \varphi_{2}\right) \mathcal{L}_{x} \\
+\left(\mathcal{D} \delta \rho \varphi_{1} s_{1}^{\beta}+\mathcal{E} \delta \rho \varphi_{2} s_{2}^{\beta}\right) \mathcal{L}_{x r} \\
\left(\mathcal{A} s_{1}^{2 \beta+1} \varphi_{1}+C \varphi_{2} s_{1}^{\beta+1} s_{2}^{\beta}\right) \mathcal{L}_{x s_{1}} \\
+\left(+C \varphi_{2} s_{1}^{\beta+1} s_{2}^{\beta}+\mathcal{B} \varphi_{2} s_{2}^{2 \beta+1}\right) \mathcal{L}_{x s_{2}}
\end{array}\right\}
\end{array}\right\}=0(10)
$$$$
\left\{\begin{array}{c}
\mathcal{L}_{t}+\mu_{1} s_{1} \mathcal{L}_{s_{1}}+\mu_{2} s_{2} \mathcal{L}_{s_{2}}+\left[r+\alpha_{1}\right] x \mathcal{L}_{x} \\
+\frac{1}{2} \mathcal{A} s_{1}^{2 \beta+2} \mathcal{L}_{s_{1} s_{1}}+\frac{1}{2} \mathcal{B} s_{2}^{2 \beta+2} \mathcal{L}_{s_{2} s_{2}} \\
+C s_{1}^{\beta+1} s_{2}^{\beta+1} \mathcal{L}_{s_{1} s_{2}}+\frac{1}{2} \delta^{2} \mathcal{L}_{r r}+\mathcal{D} \delta \rho s_{1}^{\beta+1} \mathcal{L}_{s_{1} r} \\
+\mathcal{E} \delta \rho s_{2}^{\beta+1} \mathcal{L}_{s_{2} r}+\frac{1}{2}\left(\begin{array}{c}
\alpha_{2} s_{1}^{-2 \beta} \\
+\alpha_{3} s_{2}^{-2 \beta}
\end{array}\right) \frac{\mathcal{L}_{x}^{2}}{\mathcal{L}_{x x}} \\
+\theta\left(\mu_{0}-r\right) \mathcal{L}_{r}+\left(\begin{array}{c}
\alpha_{4} \\
-\left(\mu_{1}-r\right) s_{1}
\end{array}\right) \frac{\mathcal{L}_{x} \mathcal{L}_{x s_{1}}}{\mathcal{L}_{x x}} \\
+\left(\begin{array}{c}
\alpha_{5} \\
-\left(\mu_{2}-r\right) s_{2}
\end{array}\right) \frac{\mathcal{L}_{x} \mathcal{L}_{x s_{2}}}{\mathcal{L}_{x x}}+\left(\begin{array}{c}
\alpha_{6} s_{1}^{-\beta} \\
+\alpha s_{2} s_{2}^{-\beta}
\end{array}\right) \frac{\mathcal{L}_{x} \mathcal{L}_{x x}}{\mathcal{L}_{x x}} \\
-\frac{1}{2} \mathcal{A} s_{1}^{2 \beta+2} \frac{\mathcal{L}_{x s_{1}}^{2}}{\mathcal{L}_{x x}}-\frac{1}{2} \mathcal{B s}_{2}^{2 \beta+2} \frac{\mathcal{L}_{x s_{2}}^{2}}{\mathcal{L}_{x x}} \\
-\frac{1}{2} k_{3} \rho^{2} \delta^{2} \frac{\mathcal{L}_{x x}^{2}}{\mathcal{L}_{x x}}-C s_{1}^{\beta+1} s_{2}^{\beta+1} \frac{\mathcal{L}_{x s_{1} \mathcal{L}_{x s_{2}}}}{\mathcal{L}_{x x}}
\end{array}\right\}=0(17)
$$ 
$\alpha_{1}=\frac{\mathcal{F} n\left(\mathcal{A B}-C^{2}\right)}{2 \mathcal{C}\left(2 \mu_{1} \mu_{2}-\mu_{1} r-\mu_{2} r\right)}, \alpha_{2}=\frac{\mathcal{F} \mathcal{B} \mu_{1}\left(\mu_{1}-r\right)\left(\mathcal{A B}-C^{2}\right)}{2 C\left(2 \mu_{1} \mu_{2}-\mu_{1} r-\mu_{2} r\right)}-\mathcal{G}$,

$$
\begin{aligned}
\alpha_{3} & =\frac{\mathcal{F} \mathcal{A} \mu_{2}\left(\mu_{2}-r\right)\left(\mathcal{A B}-C^{2}\right)}{2 C\left(2 \mu_{1} \mu_{2}-\mu_{1} r-\mu_{2} r\right)}-\mathcal{H}, \\
\alpha_{4} & =\frac{\mathcal{F} \mu_{1} s_{1}\left(\mu_{2}-r\right)\left(\mathcal{A B}-C^{2}\right)}{2 C\left(2 \mu_{1} \mu_{2}-\mu_{1} r-\mu_{2} r\right)}, \\
\alpha_{5} & =\frac{\mathcal{F} \mu_{2} s_{2}\left(\mu_{2}-r\right)\left(\mathcal{A B}-C^{2}\right)}{2 C\left(2 \mu_{1} \mu_{2}-\mu_{1} r-\mu_{2} r\right)}, \\
\alpha_{6} & =\frac{(\mathcal{B D}-C \mathcal{E}) \mathcal{F} \mu_{1} \delta \rho}{2 C\left(2 \mu_{1} \mu_{2}-\mu_{1} r-\mu_{2} r\right)}-k_{1} \delta \rho\left(\mu_{1}-r\right), \\
\alpha_{7} & =\frac{(\mathcal{A} \mathcal{E}-C \mathcal{D}) \mathcal{F} \mu_{1} \delta \rho}{2 C\left(2 \mu_{1} \mu_{2}-\mu_{1} r-\mu_{2} r\right)}-k_{2} \delta \rho\left(\mu_{2}-r\right)
\end{aligned}
$$

\subsection{Legendre Transformation and Dual theory}

The differential equation obtained in (17) is a non linear PDE and is somehow complex to solve. In this section, we will introduce the Legendre transformation and dual theory and use it to transform the non linear PDE to a linear PDE.

Let $f: R^{n} \rightarrow R$ be a convex function for $z>0$, define the Legendre transform

$$
N(z)=\max _{x}\{f(x)-z x\},
$$

The function $N(z)$ is the Legendre dual of the function $f(x)$. (c.f. [22])

Since $f(x)$ is convex, from Theorem 3.2 and [21], the Legendre transform for the value function $\mathcal{L}\left(t, r, s_{1}, s_{2}, x\right)$ can be defined as follows

$$
\widehat{\mathcal{L}}\left(t, r, s_{1}, s_{2}, z\right)=
$$

$$
\left.\sup \mathcal{L}\left(t, r, s_{1}, s_{2}, x\right)-z x \mid 0<x<\infty\right\} \quad 0<t<T
$$

where $\widehat{\mathcal{L}}$ is the dual of $\mathcal{L}$ and $z>0$ is the dual variable of $x$, The value of $x$ where this optimum is achieved is represented by $g\left(t, r, s_{1}, s_{2}, z\right)$, such that

$$
\begin{aligned}
& g\left(t, r, s_{1}, s_{2}, z\right)= \\
& \left(\begin{array}{c}
\inf x \mid \mathcal{L}\left(t, r, s_{1}, s_{2}, x\right) \geq z x+\widehat{\mathcal{L}}\left(t, r, s_{1}, s_{2}, z\right) \\
0<t<T .
\end{array}\right.
\end{aligned}
$$

From (20), the function $g$ and $\widehat{\mathcal{L}}$ are very much related and can be referred to as the dual of $\mathcal{L}$ and are related thus,

$$
\widehat{\mathcal{L}}\left(t, r, s_{1}, s_{2}, z\right)=\mathcal{L}\left(t, r, s_{1}, s_{2}, g\right)-z g .
$$

where

$$
g\left(t, r, s_{1}, s_{2}, z\right)=x, \mathcal{L}_{x}=z, g=-\widehat{\mathcal{L}}_{z} .
$$

Differentiating (21) with respect to $t, r, s_{1}, s_{2}$ and $x$

$$
\begin{aligned}
& \mathcal{L}_{t}=\widehat{\mathcal{L}}_{t}, \quad \mathcal{L}_{s_{1}}=\widehat{\mathcal{L}}_{s_{1}}, \mathcal{L}_{s_{2}}=\widehat{\mathcal{L}}_{s_{2}}, \quad \mathcal{L}_{r}=\widehat{\mathcal{L}}_{r},, \\
& \mathcal{L}_{r x}=\frac{-\widehat{\mathcal{L}}_{r z}}{\widehat{\mathcal{L}}_{z z}}, \mathcal{L}_{s_{1} r}=\widehat{\mathcal{L}}_{s_{1} r}-\frac{\widehat{\mathcal{L}}_{s_{1} z} \widehat{\mathcal{L}}_{r z}}{\widehat{\mathcal{L}}_{z z}}, \\
& \mathcal{L}_{S_{2} r}=\widehat{\mathcal{L}}_{S_{2} r}-\frac{\widehat{\mathcal{L}}_{s_{2} z} \widehat{\mathcal{L}}_{r z}}{\widehat{\mathcal{L}}_{z z}}, \quad \mathcal{L}_{x x}=\frac{-1}{\widehat{\mathcal{L}}_{z z}}, \\
& \mathcal{L}_{s_{1} s_{1}}=\widehat{\mathcal{L}}_{s_{1} s_{1}}-\frac{\widehat{\mathcal{L}}_{s_{1} z}^{2}}{\widehat{\mathcal{L}}_{z z}}, \mathcal{L}_{s_{2} s_{2}}=\widehat{\mathcal{L}}_{s_{2} s_{2}}-\frac{\widehat{\mathcal{L}}_{s_{2} z}^{2}}{\widehat{\mathcal{L}}_{z z}}, \\
& \mathcal{L}_{x}=z, \mathcal{L}_{s_{1} x}=\frac{-\widehat{\mathcal{L}}_{s 1 z}}{\widehat{\mathcal{L}}_{z z}}, \mathcal{L}_{S_{2} x}=\frac{-\widehat{\mathcal{L}}_{s 2 z}}{\widehat{\mathcal{L}}_{z z}} \mathcal{L}_{r r}=\widehat{\mathcal{L}}_{r r}-\frac{\widehat{\mathcal{L}}_{r z}^{2}}{\widehat{\mathcal{L}}_{z z}}
\end{aligned}
$$

At terminal time $T$, we define the dual utility in terms of the original utility function $U(x)$ as

$$
\hat{U}(z)=\sup U(x)-z x \mid 0<x<\infty\},
$$

and

$$
G(z)=\sup ?\{x \mid U(x) \geq z x+\hat{U}(z) .
$$

As a result $\widehat{\mathcal{L}}\left(t, r, s_{1}, s_{2}, z\right)=\mathcal{L}\left(t, r, s_{1}, s_{2}, g\right)-z g$.

$$
G(z)=\left(U^{\prime}\right)^{-1}(z),
$$

where $G$ is the inverse of the marginal utility $\mathrm{U}$ and note that $\mathcal{L}\left(T, r, s_{1}, s_{2}, x\right)=U(x)$.

At terminal time $T$, we can define

$$
\left.g\left(T, r, s_{1}, s_{2}, z\right)=\inf _{x>0} x \mid U(x) \geq z x+\widehat{\mathcal{L}}\left(t, r, s_{1}, s_{2}, z\right)\right\}
$$

and

$$
\widehat{\mathcal{L}}\left(t, r, s_{1}, s_{2}, z\right)=\sup _{x>0}\{U(x)-z x\}
$$

so that

$$
g\left(T, r, s_{1}, s_{2}, z\right)=\left(U^{\prime}\right)^{-1}(z) .
$$

Substituting (23) into (11), (12) and (17), we have

$$
\begin{aligned}
& \left\{\begin{array}{c}
\widehat{\mathcal{L}}_{t}+\mu_{1} s_{1} \widehat{\mathcal{L}}_{s_{1}}+\mu_{2} s_{2} \widehat{\mathcal{L}}_{s_{2}}+\left[r+\alpha_{1}\right] g z \\
+\frac{1}{2} \mathcal{A} s_{1}^{2 \beta+2} \widehat{\mathcal{L}}_{s_{1} s_{1}}+\frac{1}{2} \mathcal{B} s_{2}^{2 \beta+2} \widehat{\mathcal{L}}_{s_{2} s_{2}} \\
+\frac{1}{2} \delta^{2} \widehat{\mathcal{L}}_{r r}+\mathcal{D} \delta \rho s_{1}^{\beta+1} \widehat{\mathcal{L}}_{s_{1} r}+\mathcal{E} \delta \rho s_{2}^{\beta+1} \widehat{\mathcal{L}}_{s_{2} r} \\
-\frac{1}{2}\left(\alpha_{2} s_{1}^{-2 \beta}+\alpha_{3} s_{2}^{-2 \beta}\right) z^{2} \widehat{\mathcal{L}}_{z z} \\
-\frac{1}{2} \delta^{2}\left(1-k_{3} \rho^{2}\right) \frac{\widehat{\mathcal{L}}_{\mathcal{L}_{r}}^{2}}{\widehat{\mathcal{L}}_{z z}}+\left(\begin{array}{c}
\alpha_{4} \\
-\left(\mu_{1}-r\right) s_{1}
\end{array}\right) z \widehat{\mathcal{L}}_{s_{1} z} \\
+\left(\alpha_{5}-\left(\mu_{2}-r\right) s_{2}\right) z \widehat{\mathcal{L}}_{s_{2} z} \\
+C s_{1}^{\beta+1} s_{2}^{\beta+1} \widehat{\mathcal{L}}_{s_{1} s_{2}}+\theta\left(\mu_{0}-r\right) \widehat{\mathcal{L}}_{r} \\
+\left(\alpha_{6} s_{1}^{-\beta}+\alpha_{7} s_{2}^{-\beta}\right) z \widehat{\mathcal{L}}_{r z}
\end{array}\right\}=0 . \\
& \varphi_{1}^{*}=\left(\begin{array}{c}
-\frac{\left[\mathcal{C} s_{1}^{\beta}\left(\mu_{2}-r\right)-\mathcal{B} s_{2}^{\beta}\left(\mu_{1}-r\right)\right]}{x\left(\mathcal{A B}-C^{2}\right) s_{1}^{2 \beta} s_{2}^{\beta}} z \widehat{\mathcal{L}}_{z z}-\frac{s_{1} \widehat{\mathcal{L}}_{s_{1} z}}{x} \\
-\frac{\left(\mathcal{B D}-C^{2} \mathcal{E}\right) \delta \rho}{x\left(\mathcal{A B}-C^{2}\right) s_{1}^{\beta}} \widehat{\mathcal{L}}_{r z}
\end{array}\right) \\
& \varphi_{2}^{*}=\left(\begin{array}{c}
-\frac{\left[C s_{2}^{\beta}\left(\mu_{1}-r\right)-\mathcal{A} s_{1}^{\beta}\left(\mu_{2}-r\right)\right]}{x\left(\mathcal{A B}-C_{1}^{2} s_{1}^{\beta}{ }_{1}^{2 \beta}\right.} z \widehat{\mathcal{L}}_{z z}-\frac{s_{2} \widehat{\mathcal{L}}_{s_{2} z}}{x}-\frac{(\mathcal{A B}-\mathcal{C D}) \delta \rho}{x\left(\mathcal{A B}-\mathcal{C}^{2}\right) s_{2}^{\beta}} \widehat{\mathcal{L}}_{r z}
\end{array}\right) .
\end{aligned}
$$


From equation (22), differentiating (26), (27) and (28) with respect to $z$, we have

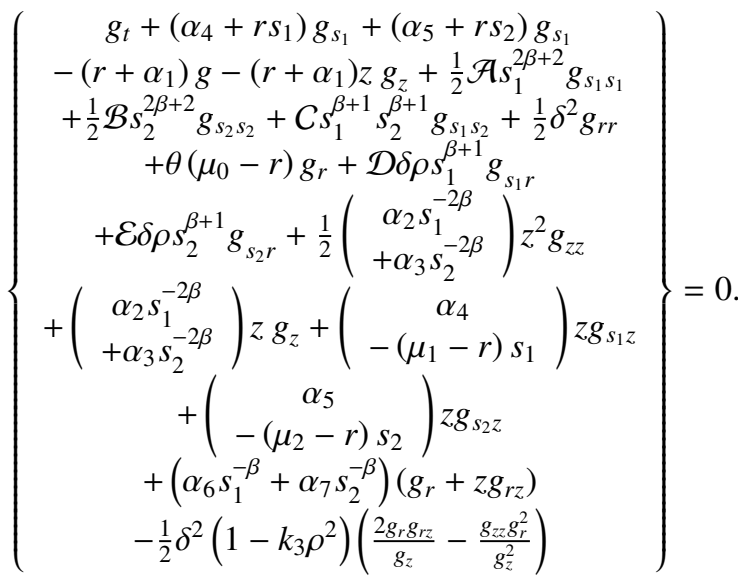

$$
\begin{aligned}
& \varphi_{1}^{*}=\left(\begin{array}{c}
\frac{\left[C s_{1}^{\beta}\left(\mu_{2}-r\right)-\mathcal{B} s_{2}^{\beta}\left(\mu_{1}-r\right)\right]}{x\left(\mathcal{A B}-C^{2}\right) s_{1}^{2 \beta} s_{2}^{\beta}} z g_{z}+\frac{s_{1}}{x} g_{s_{1}} \\
+\frac{(\mathcal{B D}-C \mathcal{C}) \delta \rho}{x\left(\mathcal{A B}-C^{2}\right) s_{1}^{\beta}} g_{r}
\end{array}\right) \\
& \varphi_{2}^{*}=\left(\begin{array}{c}
\frac{\left[C s_{2}^{\beta}\left(\mu_{1}-r\right)-\mathcal{A} s_{1}^{\beta}\left(\mu_{2}-r\right)\right]}{x\left(\mathcal{A B}-C^{2}\right) s_{1}^{\beta} s_{2}^{2 \beta}} z g_{z}+\frac{s_{2}}{x} g_{s_{2}} \\
+\frac{(\mathcal{A B}-C \mathcal{D}) \delta \rho}{x\left(\mathcal{A B}-C^{2}\right) s_{2}^{\beta}} g_{r}
\end{array}\right)
\end{aligned}
$$

where, $\mathcal{L}\left(T, r, s_{1}, s_{2}, z\right)=U(z)$ and $U(z)$ is the marginal utility of the investor. Next, we proceed to solve (29) for $g$ considering an investor with logarithm utility, then substitute the solution into (30) and (31) for the optimal investment plan using change of variable and asymptotic method.

\section{Investor's Optimal Investment Plan under Logarithm Util- ity}

Here, we consider an investor with utility function exhibiting constant relative risk aversion (CRRA) different from the one in [18] where the investor exhibited constant absolute risk aversion (CARA). Since our interest here is to determine the optimal investment plan for the investor with CRRA utility, we choose the logarithm utility function similar to the one in [20, 21].

From $[1,21]$, the logarithm utility function is given as

$$
U(x)=\ln x, x>0
$$

From (25),

$$
g\left(T, r, s_{1}, s_{2}, z\right)=\left(U^{\prime}\right)^{-1}(z)=\frac{1}{z}
$$

Next, we conjecture a solution to (21) similar to the one in [21] with the form:

$$
\left\{\begin{array}{c}
g\left(t, r, s_{1}, s_{2}, z\right)=\frac{1}{z}\left[e\left(t, r, s_{1}\right)+f\left(t, r, s_{2}\right)\right]+h(t) \\
e\left(T, r, s_{1}\right)=\frac{1}{2}, f\left(T, r, s_{2}\right)=\frac{1}{2}, h(T)=0,
\end{array}\right.
$$

$$
\left\{\begin{array}{c}
g_{t}=\frac{1}{z}\left[e_{t}+f\right]+h_{t}, \quad g_{s_{1}}=\frac{1}{z} e_{s_{1}}, g_{s_{2}}=\frac{1}{z} f_{s_{2}}, \\
g_{s_{1} s_{1}}=\frac{1}{z} e_{s_{1} s_{1}}, g_{r s_{1}}=\frac{1}{z}\left[e_{r s_{1}}+f_{r s_{1}}\right] \\
g_{s_{2} s_{2}}=\frac{1}{z} f_{s_{2} s_{2}}, g_{z}=-\frac{1}{z^{2}}[e+f], \\
g_{z z}=\frac{2}{z^{3}}[e+f], g_{s_{1} z}=-\frac{1}{z^{2}} e_{s_{1}}, g_{s_{2} z}=-\frac{1}{z^{2}} f_{s_{2}}, \\
g_{r s_{2}}=\frac{1}{z}\left[e_{r s_{2}}+f_{r s_{2}}\right], g_{r}=\frac{1}{z}\left[e_{r}+f_{r}\right], \\
g_{r r}=\frac{1}{z}\left[e_{r r}+f_{r r}\right], g_{r z}=-\frac{1}{z^{2}}\left[e_{r}+f_{r}\right]
\end{array}\right.
$$

Substituting (34) into (29), we have

$$
\left\{\begin{array}{c}
{\left[h_{t}-\left(r+\alpha_{1}\right) h\right]} \\
+\frac{1}{z}\left[\begin{array}{c}
e_{t}+\mu_{1} s_{1} e_{s_{1}}+\frac{1}{2} \mathcal{A} s_{1}^{2 \beta+2} e_{s_{1} s_{1}} \\
+\mathcal{D} \delta \rho s_{1}^{\beta+1} e_{s_{1} r}+\theta\left(\mu_{0}-r\right) e_{r}+\frac{1}{2} \delta^{2} e_{r r}
\end{array}\right] \\
+\frac{1}{z}\left[\begin{array}{c}
f_{t}+\mu_{2} s_{2} f_{s_{2}}+\frac{1}{2} \mathcal{B} s_{2}^{2 \beta+2} f_{s_{2} s_{2}} \\
+\mathcal{E} \delta \rho s_{2}^{\beta+1} f_{s_{2} r}+\theta\left(\mu_{0}-r\right) f_{r}+\frac{1}{2} \delta^{2} f_{r r}
\end{array}\right]
\end{array}\right\}=0(35)
$$

Splitting (35) we have

$$
\begin{gathered}
\left\{\begin{array}{c}
h_{t}-\left(r+\alpha_{1}\right) h=0 \\
h(T)=0
\end{array}\right. \\
\left\{\begin{array}{c}
e_{t}+\mu_{1} s_{1} e_{s_{1}}+\frac{1}{2} \mathcal{A} s_{1}^{2 \beta+2} e_{s_{1} s_{1}}+\mathcal{D} \delta \rho s_{1}^{\beta+1} e_{s_{1} r} \\
+\theta\left(\mu_{0}-r\right) e_{r}+\frac{1}{2} \delta^{2} e_{r r}=0 \\
e\left(T, r, s_{1}\right)=\frac{1}{2}
\end{array}\right. \\
\left\{\begin{array}{c}
f_{t}+\mu_{2} s_{2} f_{s_{2}}+\frac{1}{2} \mathcal{B} s_{2}^{2 \beta+2} f_{s_{2} s_{2}}+\mathcal{E} \delta \rho s_{2}^{\beta+1} f_{s_{2} r} \\
+\theta\left(\mu_{0}-r\right) f_{r}+\frac{1}{2} \delta^{2} f_{r r}=0 \\
f\left(T, r, s_{2}\right)=\frac{1}{2}
\end{array}\right.
\end{gathered}
$$

Solving equation (36) for $h$, we obtain

$$
h=0
$$

$$
g\left(t, r, s_{1}, s_{2}, z\right)=\frac{1}{z}
$$

To prove the Proposition above, we attempt to solve equations (37) and (38) by stating and proofing the following lemmas The solution of equation (37) is given as

$$
e\left(t, r, s_{1}\right)=u(t, r, m)=u_{\alpha}(t, r, m)=\frac{1}{2},
$$

where

$$
u_{\alpha}(t, r, m)=u_{1}(t, r, m)+\sqrt{\alpha} u_{2}(t, r, m)+\alpha u_{3}(t, r, m)
$$

and

$$
u_{1}(t, r, m)=\frac{1}{2}, \quad u_{2}(t, r, m)=0, \text { and } u_{3}(t, r, m)=0 .
$$

Assume

$$
\left\{\begin{array}{c}
e\left(t, r, s_{1}\right)=u(t, r, m), \quad m=s_{1}^{-2 \beta}, \\
e\left(T, r, s_{1}\right)=\frac{1}{2}
\end{array} \quad\right.
$$

then

$$
\left.\begin{array}{c}
e_{t}=u_{t}, \quad e_{s_{1}}=-2 \beta s_{1}^{-2 \beta-1} u_{m}, \\
e_{s_{1} s_{1}}=2 \beta(2 \beta+1) s_{1}^{-2 \beta-2} u_{m}+4 \beta^{2} s_{1}^{-4 \beta-2} u_{m m}, \\
e_{r}=u_{r}, \quad e_{r r}=u_{r r}, \quad e_{r s_{1}}=-2 \beta s_{1}^{-2 \beta-1} u_{r m}
\end{array}\right\}
$$


Substituting (42) into (37), we have

$$
\left[\begin{array}{c}
u_{t}-2 \mu_{1} \beta m u_{m}+\mathcal{A} \beta(2 \beta+1) u_{m} \\
+2 \beta^{2} \mathcal{A} m u_{m m}+\theta\left(\mu_{0}-r\right) u_{r} \\
+\frac{1}{2} \delta^{2} u_{r r}-2 \beta \mathcal{D} \delta \rho \sqrt{m} u_{r m}
\end{array}\right]=0
$$

We can rewrite (43) as

$$
\left(\mathcal{V}_{1}+\mathcal{V}_{2}+\mathcal{V}_{3}\right) u=0
$$

where

$$
\begin{aligned}
& \mathcal{V}_{1}=\left[\theta\left(\mu_{0}-r\right) u_{r}+\frac{1}{2} \delta^{2} u_{r r}\right] u \\
& \mathcal{V}_{2}=\left[u_{t}+\beta\left(\begin{array}{c}
\mathcal{A}(2 \beta+1) \\
-2 \mu_{1} m
\end{array}\right) u_{m}+2 \beta^{2} \mathcal{A} m u_{m m}\right] u \\
& \mathcal{V}_{3}=\left[-2 \beta \mathcal{D} \delta \rho \sqrt{m} u_{r m}\right] u
\end{aligned}
$$

Next, we follow the approach in [18] by applying the asymptotic expansion method to solve the problem in (44).

Assume that the volatility follows a slow fluctuating process, we attempt to find an asymptotic solution of (44) by a following slow-fluctuating process $r_{\alpha}$ to replace (2), in which $0<\alpha \ll 1$ is a small positive parameter:

$$
d r_{\alpha}(t)=\theta\left(\mu_{0}-r_{\alpha}(t)\right) d t-\delta d \mathcal{B}_{0}(t)
$$

Substituting (48) into (44) and also replacing $\mu_{0}-r_{\alpha}(t)$ by $\alpha\left(\mu_{0}-\right.$ $r$ ), we have

$$
\left(\alpha \mathcal{V}_{1}+\mathcal{V}_{2}+\sqrt{\alpha} \mathcal{V}_{3}\right) u_{\alpha}=0
$$

Next, we conjecture a solution for (49) as follows

$$
u_{\alpha}(t, r, m)=\left(\begin{array}{c}
u_{1}(t, r, m)+\sqrt{\alpha} u_{2}(t, r, m) \\
+\alpha u_{3}(t, r, m)
\end{array}\right) .
$$

Substituting (50) into (49),

$$
\left(\alpha \mathcal{V}_{1}+\mathcal{V}_{2}+\sqrt{\alpha} \mathcal{V}_{3}\right)\left(\begin{array}{c}
u_{1}(t, r, m) \\
+\sqrt{\alpha} u_{2}(t, r, m) \\
+\alpha u_{3}(t, r, m)
\end{array}\right)=0
$$

Simplifying the above equation, we arrive at

$$
\left(\begin{array}{c}
\mathcal{V}_{2} u_{1}(t, r, m)+\left[\begin{array}{c}
\mathcal{V}_{2} u_{2}(t, r, m) \\
+\mathcal{V}_{3} u_{1}(t, r, m)
\end{array}\right] \sqrt{\alpha} \\
+\left[\begin{array}{c}
\mathcal{V}_{1} u_{1}(t, r, m)+\mathcal{V}_{2} u_{3}(t, r, m) \\
+\mathcal{V}_{3} u_{2}(t, r, m)
\end{array}\right] \alpha
\end{array}\right)=0 .
$$

This implies that

$$
\begin{aligned}
& \left\{\begin{array}{l}
V_{2} u_{1}(t, r, m)=0 \\
u_{1}(T, r, m)=\frac{1}{2}
\end{array}\right. \\
& \left\{\begin{array}{l}
V_{2} u_{2}(t, r, m)+\mathcal{V}_{3} u_{1}(t, r, m)=0 \\
u_{1}(T, r, m)=\frac{1}{2}, u_{2}(T, r, m)=0
\end{array}\right. \\
& \left\{\begin{array}{l}
V_{1} u_{1}(t, r, m)+\mathcal{V}_{2} u_{3}(t, r, m)+\mathcal{V}_{3} u_{2}(t, r, m) \\
u_{1}(T, r, m)=\frac{1}{2}, u_{2}(T, r, m)=0 \quad u_{3}(T, r, m)=0
\end{array}\right.
\end{aligned}
$$

To obtain the solution of (50), we solve (51), (52) and (53).
Recall from (45), (46) and (47), equation (51), (52) and (53) can be expressed as

$$
\begin{gathered}
\left\{\left(\begin{array}{c}
\left.u_{1 t}+\beta\left(\begin{array}{c}
\mathcal{A}(2 \beta+1) \\
-2 \mu_{1} m
\end{array}\right) u_{1 m}+2 \beta^{2} \mathcal{A} m u_{1 m m}\right)=0 \\
u_{1}(T, r, m)=\frac{1}{2}
\end{array}\right.\right. \\
\left\{\begin{array}{c}
u_{2 t}+\beta\left(\mathcal{A}(2 \beta+1)-2 \mu_{1} m\right) u_{2 m} \\
+2 \beta^{2} \mathcal{A} m u_{2 m m}-2 \beta \mathcal{D} \delta \rho \sqrt{m} u_{1 r m}=0 \\
u_{2}(T, r, m)=0
\end{array}\right. \\
\left\{\begin{array}{c}
u_{3 t}+\beta\left(\mathcal{A}(2 \beta+1)-2 \mu_{1} m\right) u_{3 m}+2 \beta^{2} \mathcal{A} m u_{3 m m} \\
+\theta\left(\mu_{0}-r\right) u_{1 r}+\frac{1}{2} \delta^{2} u_{1 r r}-2 \beta \mathcal{D} \delta \rho \sqrt{m} u_{2 r m} \\
u_{3}(T, r, m)=0
\end{array}\right.
\end{gathered}
$$

Let

$$
u_{1}(t, r, m)=Q_{0}(t, r)+m Q_{1}(t, r)
$$

and

$$
u_{1 t}=Q_{0 t}+m Q_{1 t}, \quad u_{1 m}=Q_{1}, u_{1 m m}=0
$$

Substituting (58) in (54), we have

$$
\begin{aligned}
& Q_{0 t}+\beta \mathcal{A}(2 \beta+1) Q_{1}=0, Q_{0}(T, r)=\frac{1}{2} \\
& Q_{1 t}-2 \mu_{1} m Q_{1}=0, Q_{1}(T, r)=0
\end{aligned}
$$

Solving (60), we have

$$
Q_{1}(t, r)=0
$$

Putting (61) into (59) and solving the resultant equation, we have

$$
Q_{0}(t, r)=\frac{1}{2}
$$

Hence from (54)

$$
u_{1}(t, r, m)=Q_{0}(t, r)+m Q_{1}(t, r)=\frac{1}{2}
$$

Next, we solve (55), by assuming a solution of the form

$$
u_{2}(t, r, m)=\left(\begin{array}{c}
Q_{2}(t, r)+m^{\frac{1}{2}} Q_{3}(t, r)+m Q_{4}(t, r) \\
+m^{\frac{3}{2}} Q_{5}(t, r)
\end{array}\right)
$$

and

$$
\left\{\begin{array}{c}
u_{2 t}=Q_{2 t}+m^{\frac{1}{2}} Q_{3 t}+m Q_{4 t}+m^{\frac{3}{2}} \boldsymbol{Q}_{5 t}, \\
u_{2 m}=\frac{1}{2} m^{-\frac{1}{2}} Q_{3}+Q_{4}+\frac{3}{2} m^{\frac{1}{2}} \boldsymbol{Q}_{5} \\
u_{2 m m}=-\frac{1}{4} m^{-\frac{3}{2}} \boldsymbol{Q}_{3}+\frac{3}{4} m^{-\frac{1}{2}} \boldsymbol{Q}_{5}, \quad u_{1 r m}=0
\end{array}\right\}
$$

Substituting (65) into (55), we have

$$
\begin{gathered}
\left\{\begin{array}{c}
Q_{2 t}+\beta(2 \beta+1) \mathcal{A} Q_{4}=0 \\
Q_{2}(T, r)=0
\end{array}\right. \\
\left\{\begin{array}{c}
Q_{3 t}-\mu_{1} \beta Q_{3}+\frac{3}{2} \beta(3 \beta+1) \mathcal{A} Q_{5}=0 \\
Q_{3}(T, r)=0
\end{array}\right. \\
\left\{\begin{array}{c}
Q_{4 t}-2 \mu_{1} \beta Q_{4}=0 \\
Q_{4}(T, r)=0
\end{array}\right.
\end{gathered}
$$




$$
\left\{\begin{array}{c}
Q_{5 t}-3 \mu_{1} \beta Q_{5}=0 \\
Q_{5}(T, r)=0
\end{array}\right.
$$

Solving (66), (67), (68) and (69) with their boundary conditions, we have

$$
Q_{2}(t, r)=Q_{3}(t, r)=Q_{4}(t, r)=Q_{5}(t, r)=0,
$$

Hence from (64)

$$
u_{2}(t, r, m)=\left(\begin{array}{c}
Q_{2}(t, r)+m^{\frac{1}{2}} Q_{3}(t, r) \\
+m Q_{4}(t, r)+m^{\frac{3}{2}} Q_{5}(t, r)
\end{array}\right)=0
$$

Next, we attempt to solve (56), by assuming a solution of the form

$$
\begin{aligned}
& u_{3}(t, r, m)=\left(\begin{array}{c}
Q_{6}(t, r)+m^{\frac{1}{2}} Q_{7}(t, r)+m Q_{8}(t, r) \\
+m^{\frac{3}{2}} Q_{9}(t, r)+m^{2} Q_{9}(t, r)
\end{array}\right) \\
& u_{3 t}=Q_{6 t}+m^{\frac{1}{2}} Q_{7 t}+m Q_{8 t}+m^{\frac{3}{2}} Q_{9 t}+m^{2} Q_{10 t}, \\
& u_{3 m}=\frac{1}{2} m^{-\frac{1}{2}} Q_{7}+Q_{8}+\frac{3}{2} m^{\frac{1}{2}} Q_{9}+2 m Q_{10} \\
& u_{2 m m}=-\frac{1}{4} m^{-\frac{3}{2}} Q_{7}+\frac{3}{4} m^{-\frac{1}{2}} Q_{9}+2 Q_{10} \text {, } \\
& u_{1 r}=0, u_{1 r r}=0, u_{2 r m}=0
\end{aligned}
$$

Substituting (72) in (56), we have

$$
\begin{aligned}
& Q_{6 t}+\beta(2 \beta+1) \mathcal{A} Q_{8}=0, Q_{6}(T, r)=0 \\
& Q_{7 t}-\mu_{1} \beta Q_{7}+\frac{3}{2} \beta \mathcal{A}(3 \beta+1) Q_{9}=0, Q_{7}(T, r)=0 \\
& Q_{8 t}-2 \mu_{1} \beta Q_{8}+2 \beta \mathcal{A}(4 \beta+1) Q_{10}=0, Q_{8}(T, r)=0 \\
& Q_{9 t}-3 \mu_{1} \beta Q_{9}=0, Q_{9}(T, r)=0 \\
& Q_{10 t}-4 \mu_{1} \beta Q_{10}=0, Q_{10}(T, r)=0
\end{aligned}
$$

Solving (73), (74), (75), (4,45) and (77), we have

$$
Q_{6}(t, r)=Q_{7}(t, r)=Q_{8}(t, r)=Q_{9}(t, r)=Q_{10}(t, r)=0 .
$$

Therefore, (71) reduces to

$$
\begin{aligned}
u_{3}(t, r, m) & =Q_{6}(t, r)+m^{\frac{1}{2}} Q_{7}(t, r)+m Q_{8}(t, r) \\
& +m^{\frac{3}{2}} Q_{9}(t, r)+m^{2} Q_{9}(t, r)=0
\end{aligned}
$$

Hence, from (48), (63), (70) and (78), we have

$$
\begin{aligned}
e\left(T, r, s_{1}\right) & =u_{\alpha}(t, r, m) \\
& =u_{1}(t, r, m)+\sqrt{\alpha} u_{2}(t, r, m)+\alpha u_{3}(t, r, m)=\frac{1}{2}
\end{aligned}
$$

The solution of equation (38) is given as

$$
f\left(t, r, s_{2}\right)=v(t, r, \ell)=v_{\alpha}(t, r, \ell)=\frac{1}{2}
$$

where

$$
v_{\alpha}(t, r, \ell)=v_{1}(t, r, \ell)+\sqrt{\alpha} v_{2}(t, r, \ell)+\alpha v_{3}(t, r, \ell)
$$

and

$$
v_{1}(t, r, \ell)=\frac{1}{2}, \quad v_{2}(t, r, \ell)=0, \text { and } v_{3}(t, r, \ell)=0
$$

Assume

$$
\left\{\begin{array}{c}
f\left(t, r, s_{2}\right)=v(t, r, \ell), \quad \ell=s_{2}^{-2 \beta} \\
f\left(t, r, s_{2}\right)=\frac{1}{2}
\end{array}\right.
$$

Then

$$
\left.\begin{array}{c}
f_{t}=v_{t}, f_{s_{2}}=-2 \beta s_{2}^{-2 \beta-1} v_{\ell}, \\
f_{s_{2} s_{2}}=2 \beta(2 \beta+1) s_{2}^{-2 \beta-2} v_{\ell}+4 \beta^{2} s_{2}^{-4 \beta-2} v_{\ell \ell}, \\
f_{r}=v_{r}, \quad f_{r r}=v_{r r}, \quad f_{r s_{2}}=-2 \beta s_{2}^{-2 \beta-1} v_{r \ell}
\end{array}\right\}
$$

Substituting (80) into (38), we have

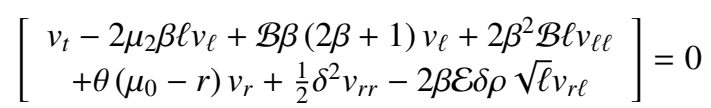

We can rewrite (81) as

$$
\left(\mathcal{M}_{1}+\mathcal{M}_{2}+\mathcal{M}_{3}\right) v=0
$$

Where

$$
\begin{aligned}
& \mathcal{M}_{1}=\left[\theta\left(\mu_{0}-r\right) v_{r}+\frac{1}{2} \delta^{2} v_{r r}\right] v \\
& \mathcal{M}_{2}=\left[\begin{array}{c}
v_{t}+\beta\left(\mathcal{B}(2 \beta+1)-2 \mu_{2} \ell v_{\ell}\right) v_{\ell} \\
+2 \beta^{2} \mathcal{B} \ell v_{\ell \ell}
\end{array}\right] v
\end{aligned}
$$

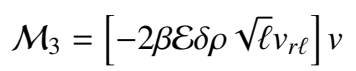

Substituting (48) into (82) and replacing $\mu_{0}-r_{\alpha}(t)$ by $\alpha\left(\mu_{0}-r\right)$, we have,

$$
\left(\alpha \mathcal{M}_{1}+\mathcal{M}_{2}+\sqrt{\alpha} \mathcal{M}_{3}\right) v_{\alpha}=0
$$

Next, we conjecture a solution for (86) as follows

$$
v_{\alpha}(t, r, \ell)=v_{1}(t, r, \ell)+\sqrt{\alpha} v_{2}(t, r, \ell)+\alpha v_{3}(t, r, \ell)
$$

Substituting (87) into (86), we have

$$
\left(\alpha \mathcal{M}_{1}+\mathcal{M}_{2}+\sqrt{\alpha} \mathcal{M}_{3}\right)\left(v_{1}(t, r, \ell)+\sqrt{\alpha} v_{2}(t, r, \ell)+\alpha v_{3}(t, r, \ell)\right)=0 .
$$

Simplifying the above equation, we arrive at

$$
\left(\begin{array}{c}
\mathcal{M}_{2} v_{1}(t, r, \ell)+\left[\mathcal{M}_{2} v_{2}(t, r, \ell)+\mathcal{M}_{3} v_{1}(t, r, \ell)\right] \sqrt{\alpha} \\
+\left[\mathcal{M}_{1} v_{1}(t, r, \ell)+\mathcal{M}_{2} v_{3}(t, r, \ell)+\mathcal{M}_{3} v_{2}(t, r, \ell)\right] \alpha
\end{array}\right)=0 .
$$

This implies that

$$
\left\{\begin{array}{c}
\mathcal{M}_{2} v_{1}(t, r, \ell)=0 \\
v_{1}(T, r, \ell)=\frac{1}{2}
\end{array}\right.
$$

$$
\left\{\begin{array}{c}
\mathcal{M}_{2} v_{2}(t, r, \ell)+\mathcal{M}_{3} v_{1}(t, r, \ell)=0 \\
v_{1}(T, r, \ell)=\frac{1}{2}, v_{2}(T, r, \ell)=0
\end{array},\right.
$$

$$
\left\{\begin{array}{c}
\mathcal{M}_{1} v_{1}(t, r, \ell)+\mathcal{M}_{2} v_{3}(t, r, \ell)+\mathcal{M}_{3} v_{2}(t, r, \ell) \\
v_{1}(T, r, \ell)=\frac{1}{2}, v_{2}(T, r, \ell)=0 v_{3}(T, r, \ell)=0
\end{array}\right.
$$

To obtain the solution of (87), we solve (88), (89) and (90). Recall from (83), (84) and (85), equation (88), (89) and (90) can be expressed as

$$
\left\{\begin{array}{c}
v_{1 t}+\beta\left(\mathcal{B}(2 \beta+1)-2 \mu_{1} \ell\right) v_{1 \ell}+2 \beta^{2} \mathcal{B} \ell v_{1 \ell \ell}=0 \\
v_{1}(T, r, \ell)=\frac{1}{2}
\end{array},\right.
$$




$$
\begin{gathered}
\left\{\begin{array}{c}
v_{2 t}+\beta\left(\mathcal{B}(2 \beta+1)-2 \mu_{2} \ell\right) v_{2 \ell} \\
+2 \beta^{2} \mathcal{B} \ell v_{2 \ell \ell}-2 \beta \mathcal{E} \delta \rho \sqrt{\ell} v_{1 r \ell}=0 \\
v_{2}(T, r, \ell)=0
\end{array}\right. \\
\left\{\begin{array}{c}
v_{3 t}+\beta\left(\mathcal{B}(2 \beta+1)-2 \mu_{2} \ell\right) v_{3 \ell}+2 \beta^{2} \mathcal{B} \ell v_{3 \ell \ell} \\
+\theta\left(\mu_{0}-r\right) v_{1 r}+\frac{1}{2} \delta^{2} v_{1 r r}-2 \beta \mathcal{E} \delta \rho \sqrt{\ell} v_{2 r \ell} \\
v_{3}(T, r, \ell)=0
\end{array} .\right.
\end{gathered}
$$

Let

$$
v_{1}(t, r, \ell)=\mathcal{R}_{0}(t, r)+\ell \mathcal{R}_{1}(t, r)
$$

and

$$
v_{1 t}=Q_{0 t}+\ell \mathcal{R}_{1 t}, \quad u_{1 \ell}=\mathcal{R}_{1}, u_{1 \ell \ell}=0 .
$$

Substituting (95) in (91), we have

$$
\begin{aligned}
& \mathcal{R}_{0 t}+\beta \mathcal{B}(2 \beta+1) \mathcal{R}_{1}=0, \mathcal{R}_{0}(T, r)=\frac{1}{2} \\
& \mathcal{R}_{1 t}-2 \mu_{1} \ell \mathcal{R}_{1}=0, \mathcal{R}_{1}(T, r)=0
\end{aligned}
$$

Solving (97), we have

$$
\mathcal{R}_{1}(t, r)=0 .
$$

Putting (98) into (96) and solving the resultant equation, we have

$$
\mathcal{R}_{0}(t, r)=\frac{1}{2} .
$$

Hence from (94)

$$
v_{1}(t, r, \ell)=\mathcal{R}_{0}(t, r)+\ell \mathcal{R}_{1}(t, r)=\frac{1}{2}
$$

Next, we solve (92), by assuming a solution of the form

$$
v_{2}(t, r, \ell)=\left(\begin{array}{c}
\mathcal{R}_{2}(t, r)+\ell^{\frac{1}{2}} \mathcal{R}_{3}(t, r) \\
+\ell \mathcal{R}_{4}(t, r)+\ell^{\frac{3}{2}} \mathcal{R}_{5}(t, r)
\end{array}\right)
$$

and

$$
\left.\begin{array}{c}
v_{2 t}=\mathcal{R}_{2 t}+\ell^{\frac{1}{2}} \mathcal{R}_{3 t}+\ell \mathcal{R}_{4 t}+\ell^{\frac{3}{2}} \mathcal{R}_{5 t}, \\
v_{2 \ell}=\frac{1}{2} \ell^{-\frac{1}{2}} \mathcal{R}_{3}+\mathcal{R}_{4}+\frac{3}{2} \ell^{\frac{1}{2}} \mathcal{R}_{5} \\
v_{2 \ell \ell}=-\frac{1}{4} \ell^{-\frac{3}{2}} \mathcal{R}_{3}+\frac{3}{4} \ell^{-\frac{1}{2}} \mathcal{R}_{5}, \quad u_{1 r \ell}=0
\end{array}\right\}
$$

Substituting (102) into (92), we have

$$
\begin{aligned}
& \mathcal{R}_{2 t}+\beta(2 \beta+1) \mathcal{B R}_{4}=0, \mathcal{R}_{2}(T, r)=0 \\
& \mathcal{R}_{4 t}-2 \mu_{2} \beta \mathcal{R}_{4}=0, \mathcal{R}_{4}(T, r)=0 \\
& \mathcal{R}_{5 t}-3 \mu_{2} \beta \mathcal{R}_{5}=0, \mathcal{R}_{5}(T, r)=0 \\
& \mathcal{R}_{3 t}-\mu_{2} \beta \mathcal{R}_{3}+\frac{3}{2} \beta(3 \beta+1) \mathcal{B R}_{5}=0, \mathcal{R}_{3}(T, r)=0
\end{aligned}
$$

Solving (103), (104), (105) and (106) with their boundary conditions, we have

$$
\mathcal{R}_{2}(t, r)=\mathcal{R}_{3}(t, r)=\mathcal{R}_{4}(t, r)=\mathcal{R}_{5}(t, r)=0,
$$

Hence from (101)

$$
v_{2}(t, r, \ell)=\left(\begin{array}{c}
\mathcal{R}_{2}(t, r)+\ell^{\frac{1}{2}} \mathcal{R}_{3}(t, r)+\ell \mathcal{R}_{4}(t, r) \\
+\ell^{\frac{3}{2}} \mathcal{R}_{5}(t, r)
\end{array}\right)=0 .
$$

Next, we attempt to solve (93), by assuming a solution of the form

$v_{3}(t, r, \ell)=\mathcal{R}_{6}(t, r)+\ell^{\frac{1}{2}} \mathcal{R}_{7}(t, r)+\ell \mathcal{R}_{8}(t, r)+\ell^{\frac{3}{2}} \mathcal{R}_{9}(t, r)+\ell^{2} \mathcal{R}_{9}(t, r)(108)$

$$
\begin{gathered}
v_{3 t}=\mathcal{R}_{6 t}+\ell^{\frac{1}{2}} \mathcal{R}_{7 t}+\ell \mathcal{R}_{8 t}+\ell^{\frac{3}{2}} \mathcal{R}_{9 t}+\ell^{2} \mathcal{R}_{10 t}, \\
u_{3 \ell}=\frac{1}{2} \ell^{-\frac{1}{2}} \mathcal{R}_{7}+\mathcal{R}_{8}+\frac{3}{2} \ell^{\frac{1}{2}} \mathcal{R}_{9}+2 \ell \mathcal{R}_{10} \\
v_{2 \ell \ell}=-\frac{1}{4} \ell^{-\frac{3}{2}} \mathcal{R}_{7}+\frac{3}{4} \ell^{-\frac{1}{2}} \mathcal{R}_{9}+2 \mathcal{R}_{10}, \\
v_{1 r}=0, \quad v_{1 r r}=0, v_{2 r \ell}=0
\end{gathered}
$$

Substituting (109) in (93), we have

$$
\begin{aligned}
& \mathcal{R}_{6 t}+\beta(2 \beta+1) \mathcal{B R}_{8}=0, \mathcal{R}_{6}(T, r)=0 \\
& \mathcal{R}_{7 t}-\mu_{2} \beta \mathcal{R}_{7}+\frac{3}{2} \beta \mathcal{B}(3 \beta+1) \mathcal{R}_{9}=0, \mathcal{R}_{7}(T, r)=0 \\
& \mathcal{R}_{8 t}-2 \mu_{2} \beta \mathcal{R}_{8}+2 \beta \mathcal{B}(4 \beta+1) \mathcal{R}_{10}=0, \mathcal{R}_{8}(T, r)=0,(112 \\
& \mathcal{R}_{9 t}-3 \mu_{2} \beta \mathcal{R}_{9}=0, \mathcal{R}_{9}(T, r)=0 \\
& \mathcal{R}_{10 t}-4 \mu_{2} \beta \mathcal{R}_{10}=0, \mathcal{R}_{10}(T, r)=0
\end{aligned}
$$

Solving (110), (111), (112), (4,82) and (114), we have

$$
\mathcal{R}_{6}(t, r)=\mathcal{R}_{7}(t, r)=\mathcal{R}_{8}(t, r)=\mathcal{R}_{9}(t, r)=\mathcal{R}_{10}(t, r)=0 .
$$

Therefore (108) reduces to

$$
\begin{aligned}
v_{3}(t, r, \ell)=\mathcal{R}_{6}(t, r) & +\ell^{\frac{1}{2}} \mathcal{R}_{7}(t, r)+\ell \mathcal{R}_{8}(t, r) \quad \\
& +\ell^{\frac{3}{2}} \mathcal{R}_{9}(t, r)+\ell^{2} \mathcal{R}_{9}(t, r)=0 .
\end{aligned}
$$

Finally, substituting (100), (107) and (115) into (87), we have

$$
\begin{aligned}
f\left(t, r, s_{2}\right) & =v_{\alpha}(t, r, \ell) \\
& =v_{1}(t, r, \ell)+\sqrt{\alpha} v_{2}(t, r, \ell)+\alpha v_{3}(t, r, \ell)=\frac{1}{2} .
\end{aligned}
$$

From Lemma 4, 4 and equation (39), Proposition 4 is proved. The optimal investment plans are given as

$$
\begin{aligned}
& \varphi_{1}^{*}=\frac{1}{x^{2} s_{2}^{\beta} s_{1}^{2 \beta}}\left[\frac{\left(\left(\sigma_{21}^{2}+\sigma_{22}^{2}\right)\left(\mu_{1}-r\right) s_{2}^{\beta}-\left(\sigma_{11} \sigma_{21}+\sigma_{12} \sigma_{22}\right)\left(\mu_{2}-r\right) s_{1}^{\beta}\right)}{\left(\sigma_{11}^{2}+\sigma_{12}^{2}\right)\left(\sigma_{21}^{2}+\sigma_{22}^{2}\right)-\left(\sigma_{11} \sigma_{21}+\sigma_{12} \sigma_{22}\right)^{2}}\right] \\
& \varphi_{2}^{*}=\frac{1}{x^{2} s_{1}^{\beta} s_{2}^{2 \beta}}\left[\frac{\left(\left(\sigma_{11}^{2}+\sigma_{12}^{2}\right)\left(\mu_{2}-r\right) s_{1}^{\beta}-\left(\sigma_{11} \sigma_{21}+\sigma_{12} \sigma_{22}\right)\left(\mu_{1}-r\right) s_{2}^{\beta}\right)}{\left(\sigma_{11}^{2}+\sigma_{12}^{2}\right)\left(\sigma_{21}^{2}+\sigma_{22}^{2}\right)-\left(\sigma_{11} \sigma_{21}+\sigma_{12} \sigma_{22}\right)^{2}}\right] \\
& \varphi_{0}^{*}=1-\varphi_{1}^{*}-\varphi_{2}^{*}
\end{aligned}
$$

Recall that equation (22) and (23) are given as

$$
\begin{gathered}
\varphi_{1}^{*}=\frac{\left[C s_{1}^{\beta}\left(\mu_{2}-r\right)-\mathcal{B s}_{2}^{\beta}\left(\mu_{1}-r\right)\right]}{x\left(\mathcal{A B}-C^{2}\right) s_{1}^{2 \beta} s_{2}^{\beta}} z g_{z}+\frac{s_{1}}{x} g_{s_{1}}+\frac{(\mathcal{B D}-C \mathcal{E}) \delta \rho}{x\left(\mathcal{A B}-C^{2}\right) s_{1}^{\beta}} g_{r} \\
\varphi_{2}^{*}=\frac{\left[C s_{2}^{\beta}\left(\mu_{1}-r\right)-\mathcal{A} s_{1}^{\beta}\left(\mu_{2}-r\right)\right]}{x\left(\mathcal{A B}-C^{2}\right) s_{1}^{\beta} s_{2}^{\beta}} z g_{z}+\frac{s_{2}}{x} g_{s_{2}}+\frac{(\mathcal{A B}-\mathcal{C D}) \delta \rho}{x\left(\mathcal{A B}-C^{2}\right) s_{2}^{\beta}} g_{r}
\end{gathered}
$$




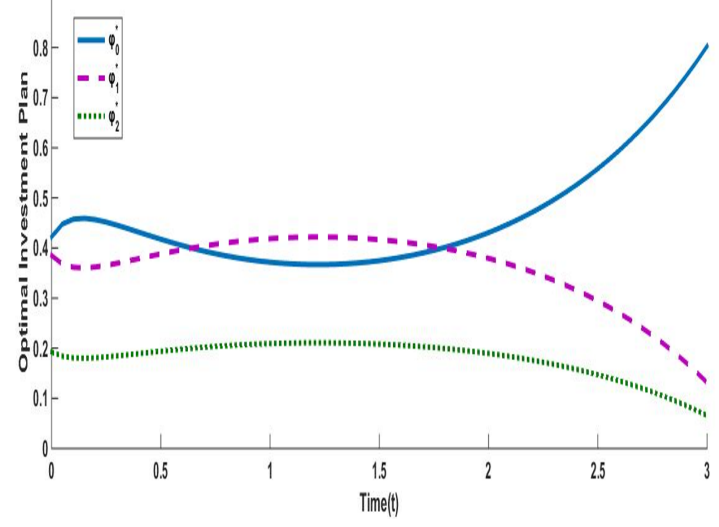

Figure 1. Time evolution of the optimal investment plan $\varphi_{0}^{*}, \varphi_{1}^{*}$, and $\varphi_{2}^{*}$.

From Proposition 4 and equation (22) we have $g\left(t, r, s_{1}, s_{2}, z\right)=\frac{1}{z}$ and $g=x$.

Differentiating $g$ with respect to $r, s_{1}, s_{2}, z$ and substitute into equation (30) and (31), we have

$$
\begin{aligned}
& \varphi_{1}^{*}=\frac{1}{x^{2} s_{2}^{\beta} s_{1}^{2 \beta}}\left[\begin{array}{c}
\left(\begin{array}{c}
\left(\sigma_{21}^{2}+\sigma_{22}^{2}\right)\left(\mu_{1}-r\right) s_{2}^{\beta} \\
-\left(\sigma_{11} \sigma_{21}+\sigma_{12} \sigma_{22}\right)\left(\mu_{2}-r\right) s_{1}^{\beta}
\end{array}\right) \\
\varphi_{2}^{*}=\frac{1}{x^{2} s_{1}^{\beta} s_{2}^{2 \beta}}\left[\begin{array}{c}
\left(\begin{array}{c}
\left(\sigma_{11}^{2}+\sigma_{12}^{2}\right)\left(\mu_{2}-r\right) s_{1}^{\beta} \\
-\left(\sigma_{11} \sigma_{21}+\sigma_{12} \sigma_{22}\right)\left(\mu_{1}-r\right) s_{2}^{\beta}
\end{array}\right) \\
\left(\sigma_{11}^{2}+\sigma_{12}^{2}\right)\left(\sigma_{21}^{2}+\sigma_{22}^{2}\right) \\
-\left(\sigma_{11}^{2} \sigma_{21}+\sigma_{12} \sigma_{22}\right)^{2}
\end{array}\right] \\
\left.\varphi_{0}^{*}=1-\varphi_{1}^{2}\right)-\left(\sigma_{11} \sigma_{21}+\sigma_{12} \sigma_{22}\right)^{2}
\end{array}\right]
\end{aligned}
$$

i. recall that from equation (33),

$$
J(t, r, s, z)=w(t, r, s)+v(t, r, s) \ln z
$$

From Proposition 4 and 4, the proof is completed.

From Lemma 4, we observed that our result is similar to the one in [3] but the difference between our result and theirs is that their interest rate is a constant while ours is stochastic.

\section{Numerical Simulations}

In this section, some numerical simulations are presented to study the effect of some sensitive parameters on the optimal investment plans under logarithm utility. To achieve this, the following data will be used unless otherwise stated;

\begin{tabular}{|l|l|l|l|l|l|l|l|}
\multicolumn{2}{|c|}{ Table 1. } \\
\begin{tabular}{|l|l|l|l|l|l|l|}
\hline \multicolumn{2}{|l|}{ Parameters } & $\sigma_{11}$ & $\sigma_{12}$ & $\sigma_{21}$ & $\sigma_{22}$ & $\mu_{1}$ \\
\hline \multicolumn{2}{|l|}{ Values } & 1.0 & 0.9 & 0.85 & 0.80 & 0.25 \\
\hline$\mu_{2}$ & $x$ & $r(0)$ & $\mathcal{S}_{1}(0)$ & $\mathcal{S}_{2}(0)$ & $\beta$ & $T$ \\
\hline 0.2 & 1 & 0.1 & 1.5 & 1.2 & -1 & 3 \\
\hline
\end{tabular}
\end{tabular}

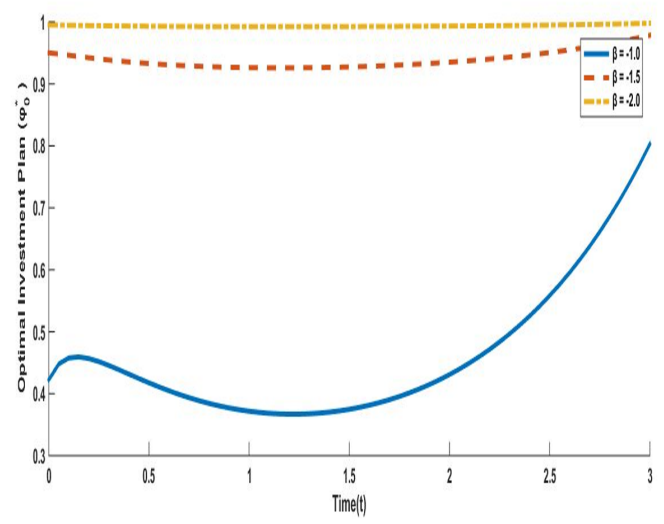

Figure 2. Time evolution of $\varphi_{0}^{*}$ with different elasticity parameter $\beta$.

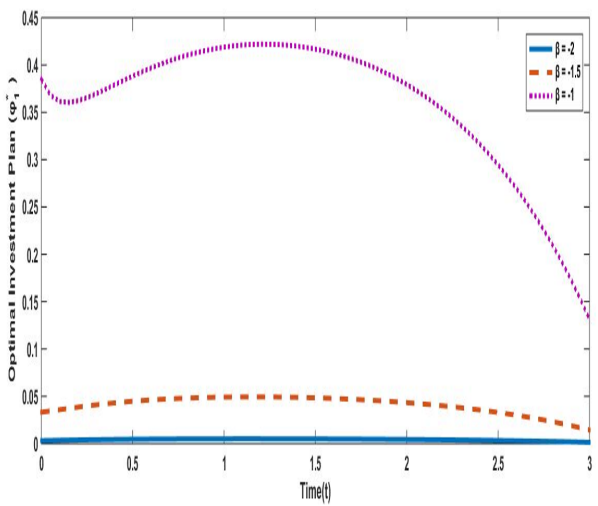

Figure 3. Time evolution of $\varphi_{1}^{*}$ with different elasticity parameter $\beta$.

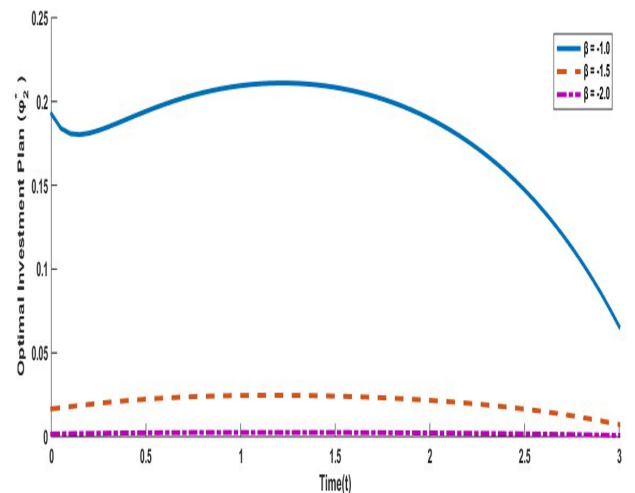

Figure 4. Time evolution of $\varphi_{2}^{*}$ with different elasticity parameter $\beta$.

\section{Discussion}

In this section, the effects of some parameters on the optimal investment plans are examined. In Figure 1, the simulation of optimal investment plans for the three assets are given against time; the graph shows that at the initial time, the investor will invest more in the risk free asset and suddenly reduce it and then increase it continually till the expiration of investment. Also, the investor will invest less in the two risky assets and suddenly 


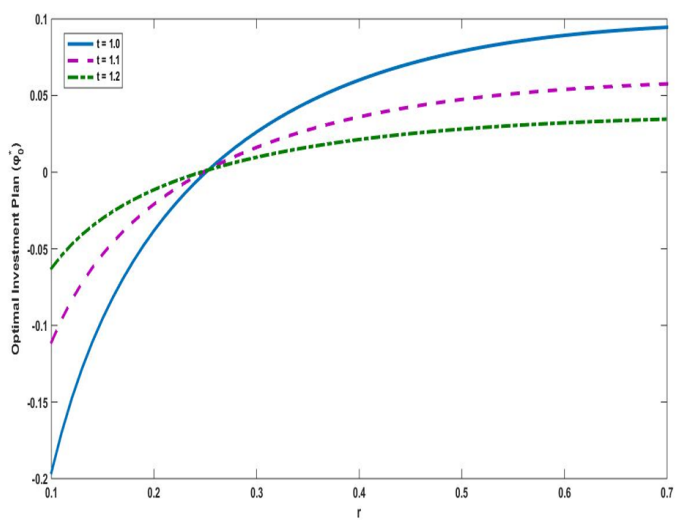

Figure 5. The effect of the risk free interest $r$ on $\varphi_{0}^{*}$.

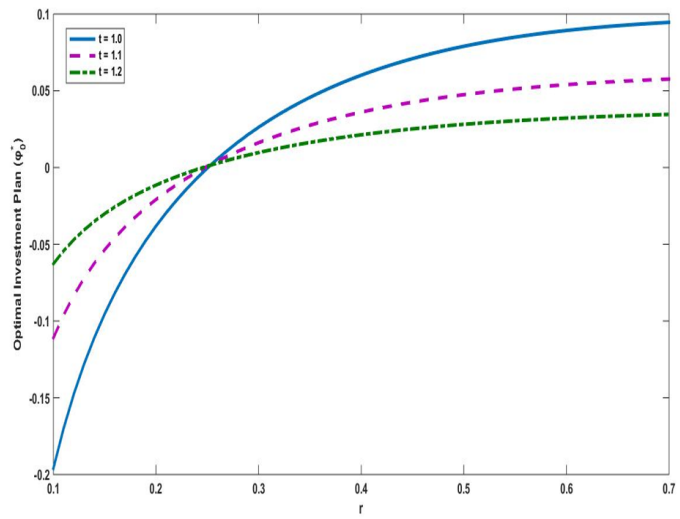

Figure 6. The effect of the risk free interest $r$ on $\varphi_{0}^{*}$.

increase it and then reduced it continually till the expiration of investment. The behavior of the optimal investment plans in Figure 1 is due to $\mathrm{O}-\mathrm{U}$ process used in modeling the risk free interest rate which reflects a fluctuation in interest rate. From this result, we can see that $\mathrm{O}-\mathrm{U}$ process enable the investor to know when there is a change in interest rate for the risk free asset thereby adjusting the optimal investment plan of the different assets to suit the market condition at each point in time. The implication of this is that each time the risk free interest rate appreciate significantly, the investor will likely to invest more in the risk free asset while reducing that of the two risky asset in a way that he or she will maximize their portfolio. Furthermore, we observed that the investor prefers investing more in stock 1 than stock 2 ; this is because the appreciation rate of stock 1 is higher than the appreciation rate of stock 2 , thereby making stock 1 more attractive than stock 2 all other factors remaining constant. Figure 2, presents simulation of $\varphi_{0}^{*}$ against time with different values of the elasticity parameter $\beta$. The graph shows that as $\beta$ reduces, the optimal investment plan $\varphi_{0}^{*}$ for the risk free asset increases. Conversely, Figures 3 and 4 , present simulations of the optimal investment plan against time with different values of the elasticity parameter $\beta$; the graphs show that as $\beta$ reduces, the optimal investment plans for the two risky assets decrease. This is because a more negative elas- ticity parameter $\beta$ gives rise to an increased probability of a large adverse movement in the stock market prices which lead to an increased expected decrease in volatility that comes with a lower $\beta$. Therefore, the investor is advised to reduce the fraction of his or her investment in the two risky assets thereby hedging the volatility risk that comes with a decreased value of $\beta$. Also, we observed that from equations (3) and (4), the stocks market prices are increasing functions of the appreciation rate $\mu_{1}$ and $\mu_{2}$ and a decreasing function of the instantaneous volatility as the elasticity parameter decreases. This implies that as $\beta$ decreases, the risky assets become less attractive thereby leading to decrease in the optimal investment plan of the two risky assets. Figure 5 and 6 give the analysis of the effect of the risk free interest rate on the optimal investment plan; in Figure 5, the optimal investment plan for the risk free asset increases as the interest rate $r$ increases. It is observed that the proportion invested in risk free assets increases faster at early of investment and very slow as retirement age gets closer; this implies that at the early stage of investment, the investor may not be interested in taking more risk but as retirement age draw closer, he invest more in the two risky assets with the aim of maximizing his portfolio. On the contrary, Figure 6 shows that the optimal investment plan for the risky assets decreases with increase in risk free interest rate $r$. This can be attributed to two factors; first, Investors are naturally attracted to high interest rate and if this happens, they will love to invest more in risk free assets for higher returns thereby reducing investment in risky assets. Secondly, considering $\left(\mu_{1}-r\right)$ in $\varphi_{1}^{*}$ where $\mu_{1}$, is the appreciation rate of stock 1 and $r$ is the risk free interest rate. We observed that as $r$ increases, $\left(\mu_{1}-r\right)$ decreases. Hence, from Figure 6, we observed that as the interest rate increases more than the appreciation rate, the investor invests less in stock 1 and when the interest rate is less than the appreciation rate, the investor invests more in stock 1 . So, in general, we observed that all other factors being constant, the investor will choose his portfolio depending on the value of the risk free interest rate and the corresponding appreciation rates of the risky assets. Finally, from Proposition 4, we observed that optimal investment plan for an investor with logarithm utility is not affected by the risk averse coefficient unlike the result in [18] where the optimal investment plan depends on the investor's behaviour towards risk.

\section{Conclusion}

In conclusion, this work studied the optimal investment plan for an investor with logarithm utility under the constant elasticity of variance model in the presence of stochastic interest rate modelled by the Ornstein-Uhlenbeck process. We developed a portfolio consisting of three assets (risk free asset and two risky assets). The Legendre transformation and dual theory with asymptotic expansion technique was used to find closed form solutions of the optimal investment plans. Also studied were the effects of some parameters on the optimal investment plans using numerical simulations. We observed that when the risk free asset is modelled by the $\mathrm{O}-\mathrm{U}$ process and the two risky asset are modelled by the CEV process, the optimal investment plan for the three assets give a fluctuation effect, showing that 
the investor's behaviour in his investment pattern changes at different time intervals due to some information available in the financial market such as the fluctuations in the risk free interest rate, appreciation rates of the price of the risky assets and the volatility of the stock market price due to changes in the elasticity parameters. Also, we observed that the investor's behavior toward risk in the case of logarithm utility does not depend on the risk averse coefficient as in the case of [18] but depend on the factors mentioned above.

\section{Acknowledgements}

The authors are very grateful to anonymous referees for their careful and diligent reading of the paper and helpful suggestions. This research has not received any sponsorship.

\section{References}

[1] D. Li, X. Rong \& H. Zhao, "Optimal investment problem with taxes, dividends and transaction costs under the constant elasticity of variance model", Transaction on Mathematics 12 (2013) 243.

[2] J. C. Cox \& S. A. Ross, "The valuation of options for alternative stochastic processes", Journal of financial economics 3 (1976) 145.

[3] J. Xiao, Z. Hong \& C. Qin, "The constant elasticity of variance (CEV) model and the Legendre transform-dual solution for annuity contracts", Insurance 40 (2007) 302.

[4] J. Gao, "Optimal portfolios for DC pension plan under a CEV model", Insurance Mathematics and Economics 44 (2009) 479.

[5] B. O. Osu, K. N. C. Njoku \& B. I. Oruh, "On the Investment Strategy, Effect of Inflation and Impact of Hedging on Pension Wealth during Accumulation and Distribution Phases", Journal of the Nigerian Society of Physical Sciences 2 (2020) 170. https://doi.org/10.46481/jnsps.2020.62

[6] M. Gu, Y. Yang, S. Li \& J. Zhang, "Constant elasticity of variance model for proportional reinsurance and investment strategies", Insurance: Mathematics and Economics 46 (2010) 580.

[7] D. Li, X. Rong, H. Zhao \& B. Yi, "Equilibrium investment strategy for DC pension plan with default risk and return of premiums clauses under CEV model", Insurance 72 (2017) 6.

[8] B. O. Osu, E. E. Akpanibah \& C. Olunkwa, "Mean-Variance Optimization of portfolios with return of premium clauses in a DC pension plan with multiple contributors under constant elasticity of variance model", International Journal of Mathematics and Compututer Science 12 (2018) 85.
[9] J. F. Boulier, S. Huang \& G. Taillard, "Optimal management under stochastic interest rates: the case of a protected defined contribution pension fund", Insurance 28 (2001) 173.

[10] G. Deelstra, M. Grasselli \& P. F. Koehl, "Optimal investment strategies in the presence of a minimum guarantee", Insurance 33 (2003) 189.

[11] J. Gao, "Stochastic optimal control of DC pension funds", Insurance $\mathbf{4 2}$ (2008) 1159.

[12] P. Battocchio \& F. Menoncin, "Optimal pension management in a stochastic framework" Insurance 34 (2004) 79.

[13] A. J. G. Cairns, D. Blake \& K. Dowd, "Stochastic life styling: optimal dynamic asset allocation for defined contribution pension plans", Journal of Economic Dynamics \&Control 30 (2006) 843

[14] C. Zhang \& X. Rong, "Optimal investment strategies for DC pension with stochastic salary under affine interest rate model”, Hindawi Publishing Corporation 2013 (2013) http://dx.doi.org/10.1155/2013/297875,

[15] E. E. Akpanibah, B. O. Osu, K. N. C. Njoku \& E. O. Akak, "Optimization of Wealth Investment Strategies for a DC Pension Fund with Stochastic Salary and Extra Contributions", International Journal of Partial Differential Equations and Applications 5 (2017) 33.

[16] K. N. C Njoku, B. O. Osu, E. E. Akpanibah \& R. N. Ujumadu, "Effect of Extra Contribution on Stochastic Optimal Investment Strategies for DC Pension with Stochastic Salary under the Affine Interest Rate Model” Journal of Mathematical Finance 7 (2017) 821.

[17] E. E. Akpanibah \& U. O. Ini, "Portfolio Strategy for an Investor with Logarithm Utility and Stochastic Interest Rate under Constant Elasticity of Variance Model", Journal of the Nigerian Society of Physical Sciences 2 (2020) 186.

[18] Y. He \& P. Chen, "Optimal Investment Strategy under the CEV Model with Stochastic Interest Rate", Mathematical Problems in Engineering 2020 (2020) 1.

[19] S. A. Ihedioha, N. T. Danat \& A. Buba, "Investor's Optimal Strategy with and Without Transaction Cost Under Ornstein-Uhlenbeck and Constant Elasticity of Variance (CEV) Models via Exponential Utility Maximization", Pure and Applied Mathematics Journal 9 (2020) 55.

[20] H. Zhao \& X. Rong, "Portfolio selection problem with multiple risky assets under the constant elasticity of variance model", Mathematics and Economics 50 (2012) 179.

[21] X. Xiao, K, Yonggui \& Kao, "The optimal investment strategy of a DC pension plan under deposit loan spread and the O-U process", (2020). Preprint submitted to Elsevier.

[22] M. Jonsson \& R. Sircar, "Optimal investment problems and volatility homogenization approximations", Modern Methods in Scientific Computing and Applications 75 (2002) 255. 